\title{
Wallerian degeneration of corticospinal tract following stroke - Indicator of worse prognosis
}

\author{
Manchikanti Venkatesh, G.H. Jhansi Priya, Amit Agrawal \\ Department of Radiology, Narayana Medical College Hospital, Nellore-524003, \\ Andhra Pradesh, India
}

A 55 year old female was brought to hospital with history of stroke 2 months earlier due to hypertension. On examination she had decreased power in left upper and lower limbs. MRI revealed chronic infarct in right fronto-parietal lobes (Figure 1) and thick linear hyperintense tract involving posterior limb of right internal capsule and right cerebral peduncle suggestive of wallerian degeneration seen after 2 months of insult. Wallerian de-

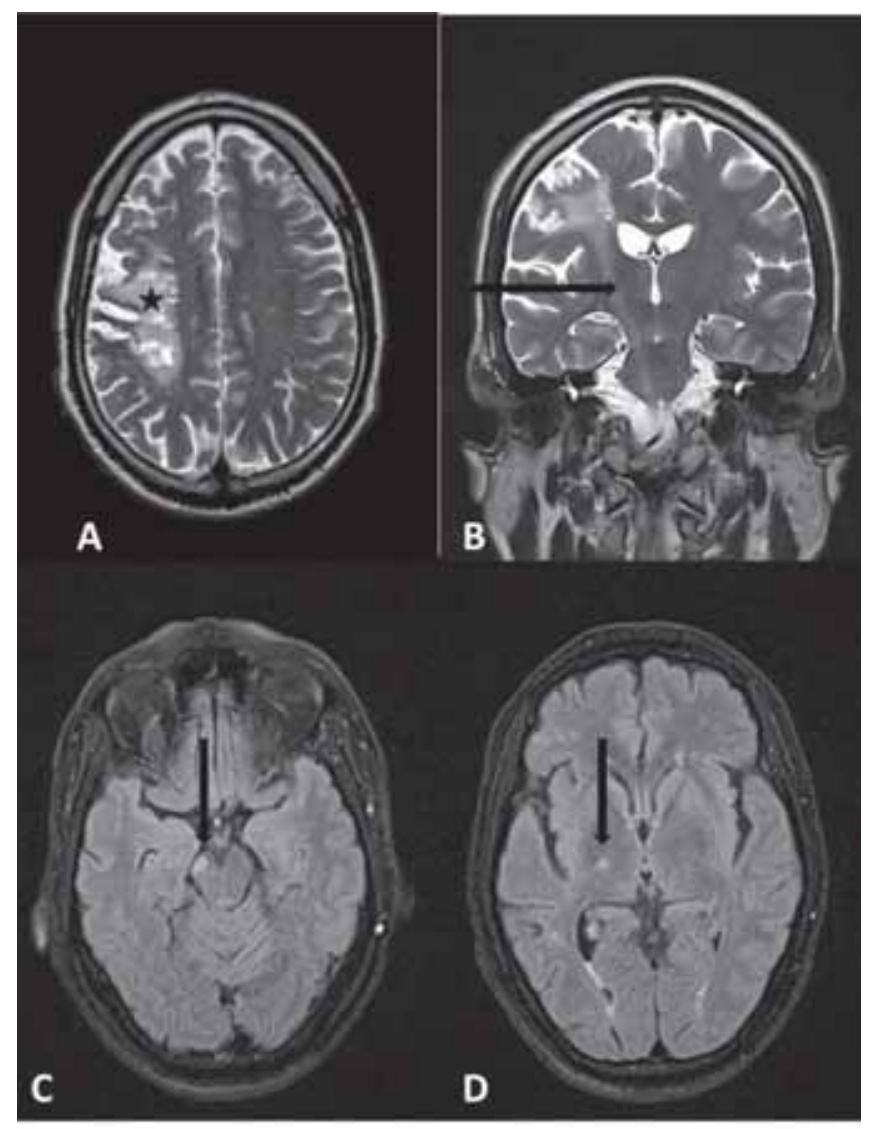

generation is a progressive anterograde disintegration of axons with demyelination after an injury/ insult. MRI is now advanced with many newer techniques like diffusion tensor imaging(DTI) to evaluate the other neuronal pathways as well. (1) Supratentorial infarct causing wallerian degeneration is a predictor of worse prognosis. Corticospinal tract integrity can be better evaluated by fractional anisotropy value of DTI (2).
FIGURE 1. MRI images of brain T2 ( $A$ and $B$ ) and FLAIR (C and D) of brain in axial plane showing chronic infarct in right fronto-parietal lobes(represented by star A) and straight hyperintensity involving the posterior limb of right internal capsule and right cerebral peduncle (represented by black arrow B,C and D). 


\section{REFERENCES}

1. Chen YJ, Nabavizadeh SA, Vossough A, Kumar S, Loevner LA, Mohan S. Wallerian Degeneration Beyond the Corticospinal Tracts: Conventional and Advanced MRI Findings. Journal of Neuroimaging. 2017 May 1;27(3):272-80.

2. Puig J, Pedraza S, Blasco G, Daunis-I-Estadella J, Prats A, Prados F, Boada I, Castellanos M, Sánchez-González J, Remollo S, Laguillo G.
Wallerian degeneration in the corticospinal tract evaluated by diffusion tensor imaging correlates with motor deficit 30 days after middle cerebral artery ischemic stroke. American Journal of Neuroradiology. 2010 Aug 1;31(7):1324-30.

Conflict of interest: none declared Financial support: none declared 